\title{
Importancia del vínculo entre las matemáticas y el Turismo
}

DOI: https://doi.org/10.33262/ap.v1i2.9

(c) (1) (9) (2)

Importance of the link between mathematics and Tourism

Dariel Armstrong Zulueta. ${ }^{1}$

\begin{abstract}
.
Currently, tourism development is supported by a whole network of studies and technologies that are based on mathematical tools and models applied to the dissimilar contexts that are generated in the sector. From the shadows they have been a key factor for interpretation, understanding and decision making in all the phenomena that can occur in tourism. The objective of this research is to demonstrate the importance of mathematics in the tourist management of a destination, from the characterization of the tools with application in tourism and their implementation, and the evaluation of the incidence of these tools in the development of the tourism management. It focuses fundamentally on the application of statistics, optimization and Information and Communication Technologies (ICT). Within ICT, he briefly characterizes the application of Big Datas and its importance for the generation of reliable information in real time. Finally, it makes a brief diagnosis of the application of databases in Cuba and its possible evolution to Big Datas.

The objectives of the research are achieved from the bibliographic consultation of different sources, where theoretical and practical cases of applications of mathematical tools are analyzed in different aspects of economic, social, financial, marketing analysis, among others.
\end{abstract}

Keywords: Tools, mathematics, tourism, statistics, optimization, ICT, Big Data.

\footnotetext{
${ }^{1}$ Universidad de La Habana. Facultad de Turismo. La Habana, Cuba. darielarmstrong@gmail.com
} 


\section{Resumen.}

En la actualidad el desarrollo turístico es soportado por toda una red de estudios y tecnologías que tienen su basamento en herramientas y modelos matemáticos aplicados a los disimiles contextos que se generan en el sector. Desde las sombras han sido un factor clave para la interpretación, comprensión y toma de decisiones en todos los fenómenos que pueden darse en el turismo. La presente investigación tiene como objetivo demostrar la importancia de las matemáticas en la gestión turística de un destino, a partir de la caracterización las herramientas con aplicación en el turismo y su implementación, y la evaluación de la incidencia de dichas herramientas en el desarrollo de la gestión turística. Se enfoca fundamentalmente en la aplicación de la estadística, la optimización y las Tecnologías de la Información y las Comunicaciones (TIC). Dentro de las TIC caracteriza brevemente aplicación de las Big Datas y su importancia para la generación de información confiable y en tiempo real. Por último, realiza un breve diagnóstico de la aplicación de las bases de datos en Cuba y su posible evolución a las Big Datas.

Los objetivos de la investigación son logrados a partir de la consulta bibliográfica de diferentes fuentes, en donde se analizan casos teóricos y prácticos de aplicaciones de herramientas matemáticas en diferentes aspectos de análisis económicos, sociales, financieros, de marketing, entre otros.

Palabras claves: Herramientas, matemáticas, turismo, estadística, optimización, TIC, Big Data.

\section{Introducción.}

El turismo se ha vuelto un factor clave en la economía de muchos países, principalmente en los que están en vías de desarrollo y ven en él una forma fundamental para incrementar su PIV. Los estudios referentes al tema lo ubican como un fenómeno social, cultural y económico, según el punto de vista desde el cual es tratado. Todos los enfoques son certeros a pesar de la variabilidad de puntos de vistas ya que fundamentalmente es un fenómeno social inherente a la voluntad humana, y por ende va con la percepción de cada región, sociedad, país, o persona que lo practica, lo estudia o lo gestiona. La claridad de la conciencia en los actores que intervienen en el fenómeno, y del rol que desempeñan durante el mismo, es lo que posibilita su correcto desarrollo ascendente en la sociedad.

La evolución que ha marcado el turismo desde su surgimiento ha seguido la velocidad de desarrollo de la humanidad misma, llevándolo a escala global y facilitando los servicios brindados para el cliente o consumidor final, que ya puede conocer todo el mundo; pero al mismo tiempo complejizando y perfeccionando todos los procesos que dan como resultado la correcta gestión del sistema. La diferenciación entre estos actores: cliente o consumidor y 
gestor, es lo que determina el nivel de complejidad percibido por la sociedad con relación al turismo.

Siguiendo este enfoque es común encontrar personas que hacen referencia al sector como el de mayor facilidad para su gestión, viéndolo desde la perspectiva del cliente el cual en menos de 30 minutos se puede gestionar unas vacaciones de excelencia del otro lado del mundo. Pero se debe tener en cuenta que detrás de esta simple acción que no depende más que del poder adquisitivo y del deseo del consumidor, se desarrolla todo un sistema turístico.

El desarrollo económico a partir de la gestión turística en cada país está ligado al nivel de competitividad que alcanza la mismo, y esto se logra no solo con la experiencia de los directivos y la confianza en el poder de atractividad de los productos turísticos; sino con estudios certeros y confiables que determinen necesidades reales de los turistas, oportunidades de desarrollo y la aplicación de las Tecnologías de la Información y las Comunicaciones (TIC).

La seriedad y confianza de estos estudios es basada en la matemática y su sinfín de aplicaciones para el desarrollo, por lo tanto, la presente investigación tiene por objetivo:

\section{Objetivo general:}

Demostrar la importancia de las matemáticas en la gestión turística de un destino.

\section{Objetivos específicos:}

- Caracterizar las herramientas matemáticas con aplicación en el turismo.

- Evaluar la incidencia de las herramientas en el desarrollo de la gestión turística.

\section{Metodología:}

El diseño de esta investigación consta de un enfoque cualitativo porque buscó comprender la aplicación de las matemáticas en el sector turístico a partir de las consultas bibliográficas.

Al respecto de este tipo de estudios Carlos Méndez afirma que:

"busca identificar características del universo de investigación, señala formas y actitudes del universo investigado, establece comportamientos concretos y descubre y comprueba la asociación entre variables". (Méndez Álvares, 2001)

Los documentos fueron parte principal desde el inicio de esta investigación, basados en ellos se pudo evaluar la incidencia real de las matemáticas en el turismo a partir de ejemplos prácticos reales o docentes en los que se realizaban distintos estudios sobre el tema.

\section{Resultados:}

\section{La estadística descriptiva:}


La estadística descriptiva ha venido siendo utilizada como una herramienta fundamental a la hora de llevar a cabo investigaciones en el área del turismo, dado que permite conocer las características de poblaciones concretas, y además realizar predicciones sobre la evolución de estas características. (Organización Mundial del Turismo (OMT), 2017)

En el caso del turismo, la estadística permite estudiar y sistematizar la evolución de la realidad turística, por ejemplo, la estacionalidad, el crecimiento, los ciclos, los impactos de la actividad, etc. También permite conocer la evolución de un determinado destino turístico valorando su gestión a corto y largo plazo. Por último, la estadística descriptiva permite analizar los errores y logros observados en el pasado con el fin de establecer políticas correctoras o potenciar las estrategias de futuro. (Organización Mundial del Turismo (OMT), 2017)

\section{Importancia de la estadística para la investigación en turismo:}

\section{Para el empresario turístico:}

- Proporciona más y mejor información sobre el sistema turístico.

- Aumenta la competitividad al permitir un mayor conocimiento del mercado y del entorno en el que se desarrolla la actividad turística.

- Reduce la incertidumbre y el tiempo de reacción ante los cambios en los requerimientos de los clientes.

- Reduce los costes generados por los errores.

\section{Para el sector público:}

- Actúa de forma más competitiva al tener mayor información.

- Es una fuente de información importante.

- Detecta la importancia del sector sobre el resto de la economía.

- Permite realizar planificaciones estratégicas.

Los datos empleados en las investigaciones turísticas tienen distintas clasificaciones en dependencia de su uso y finalidad. Principalmente se agrupan en datos de corte transversal, de series temporales y de panel.

Los Datos de corte transversal son los que se obtienen cuando, en un momento determinado del tiempo, se analizan los distintos valores de una misma variable según una serie de criterios (por ejemplo: gasto en turismo per cápita en el año 1999 para las cinco nacionalidades con mayor afluencia de turismo; pernoctaciones en el mes de agosto según categorías hoteleras; entradas vendidas en un fin de semana en un parque temático según grupos de edad; etc.). 
Una misma variable puede ser medida según diversos criterios: la evolución en el tiempo, subgrupos humanos, regiones geográficas, etc. Según el criterio que se ha establecido se pueden establecer posteriormente unas comparaciones u otras. Cuando se escoge el primer criterio (evolución temporal) se obtiene un tipo de datos conocidos como datos de series temporales, que reciben un tratamiento diferenciado del resto. (Organización Mundial del Turismo (OMT), 2017)

Los Datos de serie temporal es el conjunto de medidas de una variable de interés tomadas a lo largo del tiempo. El empleo de datos de series temporales tiene gran importancia dado que permite:

- Estudiar el comportamiento de una variable en el tiempo, es decir, si ha habido crecimiento o decrecimiento.

- Conocer la existencia de efectos estacionales.

- Distinguir si un determinado movimiento es ocasional o cíclico (y por lo tanto, es de esperar que se repita).

- Comparar la evolución de variables del turismo (pernoctaciones, gasto en turismo,

- etc.) con la de otro tipo de variables económicas (inflación, tipo de cambio, etc.), sociales (población, nivel de vida, etc.), medioambientales, etc.

Datos de panel cuando se observa la evolución de una variable en el tiempo y además se incluye algún otro tipo de criterio de comparación dentro del mismo período de tiempo, combinando, por lo tanto, la información temporal y de corte transversal.

Entre los análisis más comunes desarrollados en el turismo se encuentran los análisis de distribuciones bidimensionales.

El análisis de la realidad turística requiere muchas veces el estudio simultáneo de dos o más variables, con el objetivo de comprobar la posible relación entre ellas. Este análisis favorece el entendimiento de la realidad turística y ayuda a la toma de decisiones, tanto a nivel público como en el contexto de las empresas turísticas. Igualmente, este análisis favorece la previsión futura y la evolución de dichas variables en el tiempo.

Muchas investigaciones realizadas en el campo del turismo han presentado estudios donde se han establecido relaciones entre variables atendiendo a la experiencia y al conocimiento teórico de la realidad turística.

Así, en el análisis de la demanda de turismo se ha experimentado diferentes clases de modelos donde se ha relacionado la variable demanda, bien medida de una forma cuantitativita (número de turistas) o de una forma economicista (gasto turístico), con un conjunto de variables que han permitido explicar sus movimientos y dinamismo (Organización Mundial del Turismo (OMT), 2017). De esta forma se pueden desarrollar 
estrategias enfocadas al aprovechamiento de estas variables incidentes para favorecer el crecimiento de la demanda turística.

\section{La optimización en el turismo a partir de la programación lineal:}

Como parte del proceso de planificación de cualquier empresa resulta de vital importancia una organización que permita hacer un uso óptimo de los recursos. La optimización, implica determinar la asignación más eficiente, en el que el principal objetivo es minimizar costos y tiempo, así como maximizar las utilidades.

La programación lineal trata sobre la planeación de las actividades para obtener un resultado óptimo, esto es, el resultado que mejor alcance la meta especificada (según el modelo matemático) entre las alternativas de solución. En este caso la palabra "programación" no se refiere a programación en computadoras; sino que se le utiliza como sinónimo de planeación (Alvarado Boirivant, 2009), y es una herramienta de investigación operativa que se define como un algoritmo matemático con una función objetivo y restricciones que son formuladas a través de ecuaciones lineales que determina la asignación óptima de recursos escasos (Osorio Cuellar, 2016).

Generalmente la utilización de esta herramienta está basada en dos objetivos fundamentales a partir del modelo matemático a emplear:

1. Maximización.

2. Minimización.

En forma resumida se afirma que la programación lineal es un método matemático de resolución de problemas donde el objetivo es optimizar (maximizar o minimizar) un resultado a partir de seleccionar los valores de un conjunto de variables de decisión, respetando restricciones correspondientes a disponibilidad de recursos, especificaciones técnicas, u otras condicionantes que limiten la libertad de elección. Mediante la programación lineal se puede representar un sistema de producción a partir de un modelo o matriz en el que se incluyen (Fernández, 2015):

- Costos e ingresos generados por unidad de actividad (función objetivo).

- Aportes y requerimientos de insumos y productos por unidad de cada actividad considerada (coeficientes insumo/producto).

- Disponibilidad de recursos, especificaciones técnicas y empresariales a respetar (valores del lado derecho de las restricciones).

En concreto, permite analizar y elegir la mejor entre muchas alternativas. En términos generales se puede pensar en la programación lineal como un medio para determinar la mejor manera de distribuir una cantidad de recursos limitados en procura de lograr un objetivo expresable en maximizar o minimizar una determinada cantidad (Fernández, 2015). 
La aplicación de esta herramienta matemática se hace cada vez más necesaria en la planificación de las disímiles actividades dentro del sector turístico, entre ellas se pueden citar:

1. Aplicación en el marketing:

Conociendo todas las características de los clientes a los que está enfocada la campaña de marketing se generan una serie de restricciones referentes a la forma de llegar a cada consumidor del producto o servicio brindado, considerando, por ejemplo: variabilidad de edades, regiones geográficas, idiomas, costumbres, status social, etc. Con ellas se determina la campaña de marketing más idónea para la reducción de costos.

2. Aplicación en la restauración:

En esta parte de la prestación de servicios hoteleros y extra-hoteleros es muy variada la aplicación de este tipo de herramienta; ejemplo:

En la investigación del doctor José Fernández García, de la facultad de economía de la universidad de la habana, cuyo objetivo era el de maximizar la ganancia del restaurant sin aumentar el número de platos vendidos y manteniendo o disminuyendo el costo directo del mismo, se determinó optimización de las ganancias a partir de aplicar un modelo de programación lineal determinando como variables independientes cada uno de los alimentos de la carta menú y como restricciones el máximo número de paltos que pueden vender de cada uno. Al final el modelo determino los platos que más insidian en la rentabilidad del restaurante y los que por su resultado nulo era conveniente retirar de la misma. Estos resultados fueron comparados con otros estudios como los de ingeniería de menú y la observación del comportamiento los platos en un mes y arrojaron resultados de alta similitud, demostrando la veracidad del modelo utilizado.

3. Aplicación en el transporte aéreo:

Podemos hablar de optimización del espacio de pista consumido por cada aeronave. Esta está ligada a restricciones de tiempo en cuanto a: tiempo de revisión de la aeronave, carga de combustible, monta de pasajeros y equipaje, etc. A partir de este método se determina el uso óptimo del tiempo de cada aeronave en la pista para una mejor gestión.

4. Aplicación en el transporte terrestre (ómnibus):

La reducción de costos a partir de la optimización de combustible. Considerando como restricciones para su huso, el consumo de cada ómnibus, la cantidad de viajes, el consumo por viajes, la cantidad de ómnibus, la cantidad disponible de combustible, entre otras.

5. Aplicaciones en la gestión de servicios:

Se pude tomar por ejemplo la optimización de la cantidad de dependientes que operan en uno de los salones de un restaurante, como restricciones se tienen en cuenta la cantidad de mesas a atender por dependiente, la cantidad de mesas del salón, etc. 
6. Aplicaciones a la logística:

Este es posiblemente la parte donde la programación lineal se utilice, o se deba utilizar con mayor frecuencia y eficiencia puesto que las actividades logísticas buscan constantemente la reducción de costos, adema de tener incorporadas otras actividades de necesaria optimización como el transporte, el almacenaje, la compra y distribución de recursos.

\section{La informática y las comunicaciones.}

Hasta el momento en esta investigación se han mencionado herramientas y modelos matemáticos que facilitan la gestión de las actividades que se desarrollan en el turismo, pero en la actualidad su uso o aplicación no se realiza de forma manual. Para el desarrollo de estas actividades se ha implementado todo un mundo tecnológico basado en la informática y las comunicaciones.

Las tendencias actuales de las Tecnologías de la Información, que se manifiestan en el desarrollo prioritario de las comunicaciones y de la multimedia, vienen a satisfacerlas necesidades de información del sector turístico de una forma muy adecuada. Hablar de turismo y tecnologías de la información es referirse a las dos áreas económicas de mayor proyección para el siglo XXI. La utilización de la Tecnología de la Información y las Comunicaciones (TIC) incide en la mejora de la calidad en sus dos vertientes, por un lado, produciendo ahorro de costes y optimizando los procesos, lo que redunda en la mejora de la gestión. Por otro lado, la aplicación de estas tecnologías posibilita la prestación del servicio en mejores condiciones y la incorporación de nuevos servicios, lo que redunda en la mayor satisfacción del cliente (Instituto de Estudios Turisticos, 2012).

Una relevancia creciente ante los avances tecnológicos y su rápida adopción por la demanda que han configurado un nuevo escenario turístico observaba que el e-tourism constituía el reflejo de la digitalización de todos los procesos de la cadena de valor turística, una revolución digital que ha modificado sustancialmente la gestión turística. Incluso, la omnipresencia actual de las TIC desde el lado de la oferta (gestión y marketing, fundamentalmente) y de la demanda (información, reserva, compra y experiencia turística) diluye la diferenciación entre los procesos en línea (online) y fuera de línea (offline) (Ivars Baidal, Solsona Monzonís, \& Giner Sánchez, 2016).

Las fuerzas motrices que han provocado este cambio pueden sintetizarse en tres apartados fundamentales: la rápida evolución tecnológica, los cambios en la demanda y la búsqueda de una mayor competitividad. En una actividad intensiva en el uso de información, la generalización de Internet ha supuesto una revolución en el consumo, la producción y la comercialización turística (Ivars Baidal, Solsona Monzonís, \& Giner Sánchez, 2016).

La adopción de Internet y el desarrollo del e-commerce fueron más rápida en el turismo que en otros sectores económicos $\mathrm{y}$, a pesar de la estructura de pequeñas y medianas empresas, 
los niveles de utilización de las TIC en este ámbito son elevados (Ivars Baidal, Solsona Monzonís, \& Giner Sánchez, 2016).

La aplicación de la informática marca un crecimiento exponencial en el desarrollo del turismo $\mathrm{y}$ de su gestión. Como ejemplo se pueden tomar la implementación de software para las gestiones de recepción de un hotel, los sistemas de reservas en las agencias de viajes, los Sistemas Globales de Distribución (GDS), el desarrollo de internet y sus muchos usos como son los servicios online, las pasarelas de pago, los correos electrónicos, las redes sociales, el surgimiento de las Agencias de Viajes Online (OTA), las Big Datas y los Sistemas de Inteligencia Turística, etc. Podrían ser innumerables las aplicaciones informáticas que tributan de una forma u otra a la gestión del turismo.

Como ejemplo se tomará el uso de las Big Datas como herramienta informática para la gestión de empresas y destinos turísticos.

Cuando se habla de Big Data el termino es referido a conjuntos de datos o combinaciones de conjuntos de datos cuyo tamaño (volumen), complejidad (variabilidad) y velocidad de crecimiento (velocidad) dificultan su captura, gestión, procesamiento o análisis mediante tecnologías y herramientas convencionales, tales como bases de datos relacionales y estadísticas convencionales o paquetes de visualización, dentro del tiempo necesario para que sean útiles (Power Data, 2020).

La naturaleza compleja del Big Data se debe principalmente a la naturaleza no estructurada de gran parte de los datos generados por las tecnologías modernas, como los web logs, la identificación por radiofrecuencia (RFID), los sensores incorporados en dispositivos, la maquinaria, los vehículos, las búsquedas en Internet, las redes sociales como Facebook, computadoras portátiles, teléfonos inteligentes y otros teléfonos móviles, dispositivos GPS y registros de centros de llamadas.

El análisis de toda la amplia red de datos que se genera en una ciudad, en un país o en una región determinada es lo que le da la verdadera vida funcional a las big datas. El desarrollo de algoritmos de análisis determina información crucial en el estudio del turismo.

Todo lo anteriormente hablado en esta investigación se recoge y se lleva a la actualidad a través de softwares que se encargan del procesamiento y análisis de toda la información que pueda ser obtenida en un destino turístico.

Lo importante no es que la empresa o destino disponga de estos datos, sino que los trabaje para obtener información de valor. La información obtenida facilitará la toma de decisiones predictivas, $y$, por ende, generará un impacto positivo en los resultados de la compañía o destino gracias a la puesta en marcha de acciones adaptadas a las necesidades reales del público objetivo. 


\section{Aplicación de las Big Datas en Cuba:}

En Cuba a partir de 1997 se realizan las primeras instalaciones de un software de origen portugués NewHotel. Con un diseño novedoso fue el primer sistema instalado que usaba a plenitud las bondades y facilidades del entorno Microsoft Windows y una poderosa base de datos en Oracle. Este llegó a emplearse en alrededor de 30 hoteles y unos 15 restaurantes. Sin duda NewHotel fue el sistema que más mostró avances en su época, por el amplio diapasón de sus soluciones y la novedad que incorporó a las mismas (Vázquez Alfonso, 2018).

Posteriormente surgió Zun, un desarrollo más moderno, con una concepción totalmente nacional, además de pasar a un ambiente moderno y con MySQL como gestor de base de datos. Zun mejoró significativamente su apariencia y funcionalidad respecto a NewHotel, llegando a ser el Sistema de Gestión Hotelera (SGH) de mayor presencia en las instalaciones hoteleras de Cuba. Los SGH son sistemas estratégicos para las cadenas cubanas y el MINTUR, pues la información que registran constituye la fuente de gran parte de la estadística general sobre los arribos de turistas, sus gustos, los aspectos de calidad, estudios de segmentación, satisfacción, repitencia, reacciones a las rebajas, comportamiento de las Agencias del MINTUR, Cuentas por Cobrar internacionales; aspectos de los que se ocupa el MINTUR (Vázquez Alfonso, 2018).

En la actualidad la demanda de servicios turísticos se encuentra creciendo y de ahí que las organizaciones turísticas cubanas tengan un papel fundamental en la gestión de la información turística ofrecida al usuario final con el objetivo de aumentar la calidad del sector turístico de nuestro país. La información que se gestiona en estas entidades es de suma importancia para la organización, sin embargo, se observa que no se realiza un correcto manejo de la misma, ya no se apoyan en las ventajas que ofrecen las nuevas tecnologías (Vázquez Alfonso, 2018).

A pesar de que el país cuenta con sistemas informáticos para la captación de la información generada en los hoteles, en las agencias de viajes, y alguno de los restaurantes, esta no basta para hablar de Big Data en Cuba. Estos sistemas generan bases de datos de carácter local, para su uso en el establecimiento al que pertenezca. Las mismas son aprovechadas por las instituciones que monitorean el turismo en la zona o el destino al que pertenezcan, arribando a generalidades a partir de estos casos particulares.

Para poder implementar sistemas de información Big Data es necesario:

- Extender la implementación de estos sistemas locales a todas las entidades vinculadas al sector turístico.

- Modernizar constantemente los softwares empleados en la generación de las informaciones en tiempo real.

- Capacitar profesionales para que se desempeñen como científicos de datos. 
- Diseñar una base de datos multidimensional que logre vincular toda la información de los servicios turísticos.

- Implementar el banco de datos para el monitorio y toma de decisiones en el sector turístico.

El desarrollo de esta base de datos se debe realizar de manera gradual; monitoreando los resultados que genera para poder corregir los errores que puedan ser visualizados. La implementación debe hacer inicialmente por zonas y destinos turísticos, con el objetivo de ir vinculando todos los servicios dados, y las opiniones generadas en cada uno de ellos, hasta lograr integraciones de Big Data a niveles provinciales, y nacional.

\section{Conclusiones.}

- La presente investigación permitió arribar a las siguientes conclusiones:

- Las herramientas permiten conocer las características de poblaciones concretas, y además realizar predicciones sobre la evolución de estas características.

- La aplicación de modelos matemático es extensiva a todas las actividades dentro del sector turístico.

- El uso de las TIC marca el desarrollo de los destinos turísticos elevando su competitividad.

- Es necesaria la aplicación de sistemas de análisis de grandes volúmenes de información en tiempo real en el destino Cuba para la mejor gestión turística.

\section{Referencias bibliográficas.}

Alvarado Boirivant, J. (29 de enero de 2009). La Programacion Lineal, aplicacion de las pequeñas y medianas empresas. Obtenido de Reflexiones: http://www.redalyc.org/articulo.oa?id=72912559007

Fernández, G. (2015). Un Modelo de programacion lineal para la optimizacion de la ganancia en un estaurant y su comparacion con otras tecnicas utilizadas de perfeccionamiento del menu. La Habana: Univercidad de la Habana.

Guevara, A., Aguayo, M., Aguayo, A., \& Araque, F. (2013). Informática aplicada al turismo. Ediciones Pirámide, 312.

Instituto de Estudios Turisticos. (2012). Tegnologia de la informacion en el turismo. Madrid, España: Secretaría de Estado de Comercio, Turismo y Pymes.

Ivars Baidal, J., Solsona Monzonís, J., \& Giner Sánchez, D. (2016). Gestión turística y tecnologías de la información. Documents d'Anàlisi Geogràfica 2016, 327-346. 
Méndez Álvares, C. E. (2001). Metodología. Diseño y desarrollo del proceso de investigación., 137.

Millán Gasca, A. (2006). La aplicación de las Matematicas a los problemas de administración y control: Antecedentes Históricos. ILUIL, vol.26, 929-961.

Organización Mundial del Turismo (OMT). (26 de mayo de 2017). Apuntes de Metodología de la Investigación en el Turismo. Obtenido de e-unwto.org: http://www.eunwto.org/doi/book/10.18111/9789284404889 - Friday, May 26, 2017 7:39:52 PM Secretaría de Turismo IP Address:189.204.93.100

Osorio Cuellar, P. B. (2016). Programación lineal para la distribución de viajes en. Lima: Universidad Nacional de San Marcos, Facultad de Ciencias Matemáticas.

Power Data. (29 de enero de 2020). Power data. Obtenido de Power data: https://www.powerdata.es/big-data

Vázquez Alfonso, Y. (2018). Banco de datos turísticos para el monitoreo y toma de decisiones en entidades del Turismo. La Habana: Facultad de Turismo.

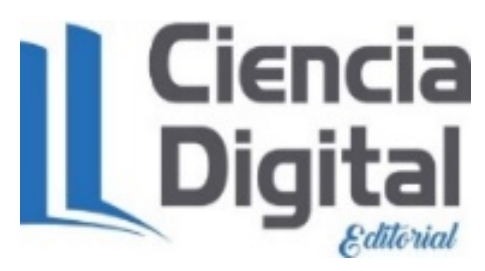




\section{Para citar el artículo indexado}

Armstrong Zulueta, D. (2020). Importancia del vínculo entre las matemáticas y el Turismo. AlfaPublicaciones, 1(2), 17-29. https://doi.org/10.33262/ap.v1i2.9

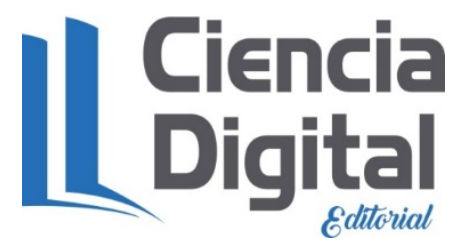

El artículo que se publica es de exclusiva responsabilidad de los autores y no necesariamente reflejan el pensamiento de la Revista Alpha Publicaciones.

El artículo queda en propiedad de la revista y, por tanto, su publicación parcial y/o total en otro medio tiene que ser autorizado por el director de la Revista Alpha Publicaciones.
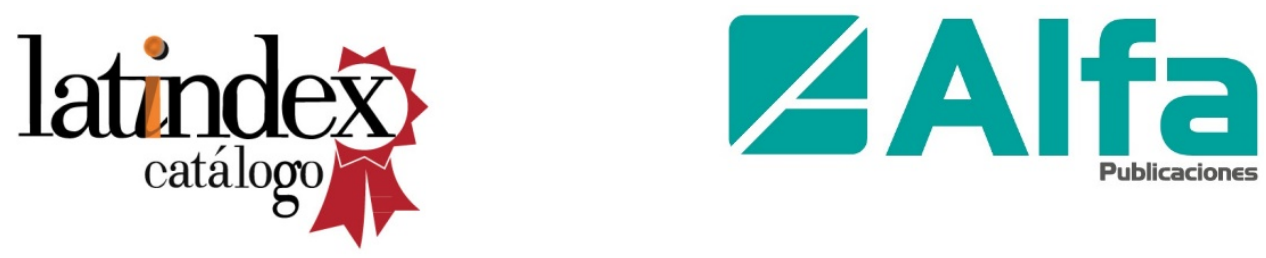